\title{
TAGUNG
}

\section{Prioritätenwechsel in der EU-Finanzpolitik? Die Agenda 2007 im Zeichen der Lissabon-Agenda}

\author{
Philipp Hessel*
}

Die Debatte über die zukünftige Finanzielle Vorausschau für den Zeitraum 2007 bis 2013 stand - nach dem Kompromiss über den Stabilitäts- und Wachstumspakt - ganz oben auf der Agenda der luxemburgischen Ratspräsidentschaft. Allerdings haben sich die Verhandlungen über die Einkünfte- und Ausgabenobergrenzen zu einer heftigen Auseinandersetzung zwischen der Europäischen Kommission und mehreren Nettozahlern entwickelt. Auch die Vermittlungsversuche der luxemburgischen Ratspräsidentschaft blieben bis Anfang Juni ohne Ergebnis. Dabei knüpft die Debatte über den zukünftigen Haushaltsplan der Europäischen Union nahezu nahtlos an die Diskussion über die Lissabon-Agenda unter niederländischer Ratspräsidentschaft an. Diesbezüglich verdeutlichte jüngst der so genannte Kok-Bericht ${ }^{1}$ erneut die Defizite der Europäischen Union im Bereich der Wettbewerbsfähigkeit und Wachstumsdynamik. Nach der eher nüchtern ausgefallenen ,Lissabon'-Halbzeitbilanz schienen sich alle Beteiligten darüber einig zu sein, dass die Strategie dringend einer Wiederbelebung bedürfe, um nicht gänzlich hinter ihren Ansprüchen zurückzubleiben. Als Konsequenz verfolgt die Kommission das Ziel, den Reformprozess durch eine deutlichere Schwerpunktsetzung neu zu beleben. Somit liegt die Frage nahe, was der mittelfristige Haushaltsplan der Europäischen Union zum Erreichen der so genannten Lissabon-Ziele beitragen kann und inwieweit sich diese Ziele in der Struktur des

\begin{tabular}{|l|}
\hline \multicolumn{1}{|c|}{ Prioritätenwechsel in der } \\
EU-Finanzpolitik? Die Agenda 2007 \\
im Zeichen der Lissabon-Agenda \\
Interdisziplinäre Tagung des Arbeitskreises \\
Europäische Integration in Zusammenarbeit mit der \\
Universität Trier und dem Zentrum für Europäische \\
Wirtschaftsforschung, Mannheim mit Unter- \\
stützung der Europäischen Kommission und der \\
$\quad$ ASKO EUROPA-STIFTUNG \\
$\quad$ Europäische Akademie Otzenhausen, \\
$\quad$ 14.-15. April 2005 \\
Wissenschaftliche Leitung: \\
Dr. Friedrich Heinemann, ZEW Mannheim \\
Prof. Dr. Joachim Schild, Universität Trier \\
Begrüßung und Einführung \\
Prof. Dr. Joachim Schild \\
Die Kommissionsvorschläge und die \\
nationale Haushaltspolitik \\
Dr. Carsten Pillath, Bundesministerium der \\
Finanzen, Berlin \\
Dr. Christian Weise, Europäische Kommis- \\
sion, Brüssel \\
Dr. Friedrich Heinemann, ZEW Mannheim \\
Reform des Eigenmittelsystems der \\
Gemeinschaft \\
Peter Becker, Stiftung Wissenschaft und \\
Politik, Berlin \\
Peter Schönberger, Europäischer Rech- \\
nungshof, Luxemburg \\
Prof. Dr. Rolf Caesar, Universität Hohenheim \\
\hline
\end{tabular}

\footnotetext{
* Philipp Hessel, studentischer Mitarbeiter am Lehrstuhl für vergleichende Regierungslehre von Prof. Dr. Schild an der Universität Trier.

1 Wim Kok: Die Erweiterung der Europäischen Union: Errungenschaften und Herausforderungen, Bericht an die Europäische Kommission, Brüssel 2003, abrufbar unter: http://80.237.230.157/download/pdf/kok_report. de.pdf (letzter Zugriff 14.06.05).
} 
zukünftigen Haushalts niederschlagen werden. Antworten auf diese Frage versuchten die Teilnehmer und Referenten auf der vom Arbeitskreis Europäische Integration in $\mathrm{Zu}$ sammenarbeit mit der Universität Trier sowie dem Mannheimer Zentrum für Europäische Wirtschaftsforschung (ZEW) mit finanzieller Unterstützung der ASKO Europa-Stiftung veranstalteten Tagung zu finden. Sie fand also vor den beiden Verfassungsreferenden - und mit Blick auf die Position der Bundesregierung und der Ankündigung von Neuwahlen sowie dem Treffen des Europäischen Rats zum Abschluss der luxemburgischen Präsidentschaft statt.

Joachim Schild oblag es, in die Thematik einzuführen. Er betonte, dass die Umsetzung der Lissabon-Agenda zunächst einmal Sache der Mitgliedstaaten sei. Dabei habe die Europäische Kommission in erster Linie die Aufgabe der Politikkoordinierung. Dadurch stelle sich auch erst in zweiter Linie die Frage, inwieweit der Haushalt der Europäischen Union zur Umsetzung der Lissabon-Agenda beitragen kann. Nichtsdestotrotz erscheine es grundsätzlich wichtig, nach dem Zusammenhang zwischen Erreichen der Lissabon-Ziele und dem zukünftigen Haushalt der Europäischen Union zu fragen, gehe es für den Zeitraum von 2007 bis 2013, laut dem Vorschlag der Kommission, doch immerhin um eine Summe von rund 1.025 Milliarden Euro an Verpflichtungsermächtigungen und 900 Milliarden Euro an Zahlungsermächtigungen. Der Einsatz dieser öffentlichen Gelder sollte sich dabei an den Herausforderungen und Prioritäten für das Handeln der Europäischen Union messen lassen. Ein unbefangener Beobachter vom Mars, so Schild, würde im Unionshaushalt wohl zunächst nur wenig entdecken, was dem Erreichen der Lissabon-Ziele und besonders dem Ziel verbesserter Wettbewerbsfähigkeit diene. Weiterhin finde sich auch im so genannten Sapir-Bericht ${ }^{2}$ die nüchterne Feststellung, dass der Haushalt der Europäischen

\section{Reform der Strukturpolitik: ein Beitrag zur EU-Wettbewerbsfähigkeit?}

Prof. Dr. Ingeborg Tömmel, Universität Osnabrück

Thomas Wobben, Verbindungsbüro des Landes Sachsen-Anhalt bei der EU, Brüssel

Lissabon-Agenda und Agenda 2007

Dr. Georg Licht, ZEW Mannheim

Dr. Pascal Hector, Auswärtiges Amt, Berlin Raoul Wirtz, Außenministerium des Großherzogtums Luxemburg

Union ein historisches Relikt sei, das mit dem heutigen und künftigen Stand das Integration nicht konsistent sei. Betrachte man den Vorschlag der Kommission, so sei dieser eher als konservativ zu bewerten, nehme er doch an der derzeitigen Struktur der Haushaltsentwicklung keine dramatischen Änderungen vor. Dies sei jedoch zu einem großen Teil auf die Kenntnis der Kommission über die Standpunkte der meisten Mitgliedsländer sowie die Erfahrungen aus den vergangenen Verhandlungen zurückzuführen. Allerdings seien auch einige Versuche sichtbar, Akzente für das Erreichen der Lissabon-Ziele zu setzen. Zu nennen seien diesbezüglich zunächst die Stabilisierung der Agrarausgaben und eine Reduzierung ihres Anteils am Gesamthaushalt sowie die betonte Konzentration der Kohäsionspolitik auf eine begrenzte Anzahl von Gemeinschaftsprioritäten. Darüber hinaus falle die deutliche Erhöhung der Mittelansätze für die Forschungs- und Technologiepolitik und die drastische Erhöhung der Mittel für transeuropäische Netze auf. Eine deutliche Steigerung der Mittelansätze könne man im Vorschlag der Kommission auch für die allgemeine und berufliche Bildung und den sehr umstrittenen und mittlerweile nicht mehr auf dem Tisch befindlichen Vorschlag für einen Wachstumsfonds beobachten.

2 André Sapir/Philippe Agnion/Giuseppe Bertola/Martin Hellwig/Jean Pisami-Ferry/Dariusz Rosati/José Viñals/ Helen Wallace: An Agenda for a Growing Europe, Bericht an die Europäische Kommission, Oxford 2004, auch abrufbar unter: http://www.euractiv.com/ndbtext/innovation/sapirreport.pdf (letzter Zugriff 14.06.05). 
Die Agenda 2007-Verhandlungen könnten dabei zur ersten schweren Bewährungsprobe der erweiterten Europäischen Union werden. Beim Vergleich der laufenden Verhandlungen mit vorherigen Verhandlungssituationen würden deutliche Unterschiede auffallen. So fänden die derzeitigen Verhandlungen in einem veränderten politischen und wirtschaftlichen Kontext statt, der seinen Ausdruck in der gewachsenen Mitgliederzahl, der Heterogenität ihrer wirtschaftlichen Situation und Dynamik, aber natürlich auch in der deutlich verringerten Finanzierungsbereitschaft der bisherigen Nettozahler finde. So sei es 1988 beim so genannten Delors-I-Paket noch möglich gewesen, eine Erhöhung der Haushaltsmittel zu Gunsten europäischer Integrationsprojekte durchzusetzen, da diejenigen Staaten, die beispielsweise die Verwirklichung des Binnenmarkts vorantreiben wollten, noch die Bereitschaft $\mathrm{zu}$ Kompensationszahlungen $\mathrm{zu}$ Gunsten wirtschaftlich schwächerer Staaten besessen hatten. Heute scheine ein ähnliches integrationspolitisches Großprojekt, zu dessen Gunsten die Nettozahler bereit wären, ihre Geldbörse zu öffnen, nicht in Sicht zu sein. Ein wichtiges Teilbudget, das Agrarbudget, sei darüber hinaus bereits festgezurrt, so dass sich die Verhandlungsdynamik auf andere Haushaltsposten konzentriere. Aus diesem Grund befürchtete Schild, dass genau die Haushaltsrubriken gekürzt werden könnten, die zur Verwirklichung der Lissabon-Agenda von Nöten wären, namentlich die Forschungsund Technologiepolitik sowie die Kohäsionspolitik.

\section{Der Zusammenhang zwischen nationaler Haushaltspolitik und dem Unionshaushalt}

$\mathrm{Zu}$ Beginn seines Vortrags wies Carsten Pillath darauf hin, dass er gerne hinter der zweiten Überschrift des Tagungstitels ebenfalls ein Fragezeichen gesehen hätte. Er empfahl in diesem Zusammenhang die Lektüre der Lissabon-Schlussfolgerungen des Jahres 2000, da diese aus gutem Grund lediglich ganz geringe Referenzen zum EU-Budget enthielten. So tauche der Zusammenhang de facto erstmals mit dem Vorschlag der Kommission vom Februar 2004 auf. Damit würde die LissabonAgenda als wunderbarer Legitimationsgrund für zusätzliche Mittelaufwendungen herhalten. Nachdem die ländliche Entwicklung laut Kommissionsvorschlag ein zentraler Bestandteil der Lissabon-Agenda sei, bleibe nur noch abzuwarten, bis auch die letzte der fünf Haushaltsrubriken, nämlich die Verwaltungsausgaben, dieser ebenfalls zugeordnet werden würde.

Zum Grundverständnis der Debatte um die Lissabon-Agenda und den EU-Haushalt erklärte Pillath, dass es wichtig sei, die Diskussion in dem Dreieck von Haushaltserfordernissen auf europäischer Ebene, der LissabonAgenda sowie der nationalen Haushaltskonsolidierung zu betrachten. So sei nicht völlig von der Hand zu weisen, dass die Forderung nach einer Festschreibung der Bruttonationaleinkommen-Abführungen auf ein Prozent sicherlich eine kommunikative Komponente aufweise, dies aber nicht den Kern der Argumentation darstelle. Vielmehr sei vor dem Hintergrund der Konsolidierungsbemühungen in Deutschland und den eindeutigen Empfehlungen der Kommission, keine Steuererhöhungen durchzuführen, eine Ausgabensenkung eine logische Konsequenz. Auch in den Gesprächen mit anderen Mitgliedstaaten hätte sich zunehmend herauskristallisiert, dass die Bruttoabgaben als eigentlicher Lastindikator für die Mitgliedstaaten zu begrenzen seien. Dadurch sei auch sehr schnell klar geworden, dass man auf ein Ziel hinsteuern würde, das eine Begrenzung um den relativen Anteil Status quo von etwa einem Prozent festschreibt. Dies habe entgegen der Argumentation der Kommission und einiger Mitgliedstaaten jedoch nichts mit einem Einfrieren des Haushalts zu tun.

Zum Verständnis der Größenordnung führte Pillath aus, dass die Bundesrepublik Deutschland gegenwärtig rund 22 Milliarden Euro jährlich in den EU-Haushalt einzahle. Würde man im Kommissionsvorschlag, entgegen dem gegenwärtigen Zahlenwerk, die Inflation 
berïcksichtigen, so stiege am Ende der kommenden Periode der durchschnittliche jährliche Beitrag der Bundesrepublik auf über 35 Milliarden Euro an. Auch bei einer Obergrenze von einem Prozent würde eine Zusatzbelastung von zirka 6,2 Milliarden Euro jährlich für Deutschland hinzukommen. Die Frage laute somit nicht, Einfrieren der Zahlungen oder nicht, sondern in welcher Höhe die Bruttoabführungen ansteigen werden. Erschwerend komme hinzu, dass nicht geklärt sei, wie die Ermahnung zur nationalen Haushaltskonsolidierung und die Aufforderung zu einer Erhöhung der Abführungen an den Haushalt der Europäischen Union zusammenpassen.

Das Zeitfenster für eine Einigung sei durch die anstehenden Wahlen im Vereinigten Königreich und das französische Referendum extrem knapp. Sollte es tatsächlich zunächst zu keiner Lösung kommen, stelle dies jedoch keineswegs den Zusammenbruch Europas dar. Angesichts der schwierigen Verhandlungsposition des Vereinigten Königsreich sei es jedoch unwahrscheinlich, dass unter britischer Ratspräsidentschaft ein Kompromiss erreicht werden könne, wodurch alles auf die österreichische Ratspräsidentschaft hinauslaufen würde. Grundsätzlich zeigte sich Pillath jedoch optimistisch, dass eine Lösung gefunden werde, mit der die Europäische Union funktionieren und arbeiten könne.

Christian Weise erläuterte zunächst den Vorschlag der Kommission zur nächsten Finanziellen Vorausschau 2007 bis 2013. So zeigte er auf, dass die Mittel der Ausgabenkategorie 1a „Wettbewerbsfähigkeit für Wachstum und Beschäftigung", also die so genannte Lissabon-Linie, nach dem Vorschlag der Europäischen Kommission in etwa auf das Doppelte steigen werden. Mittels der Ausgabenkategorie $1 \mathrm{~b}$ „Kohäsion für Wachstum und Beschäftigung" solle bis etwa zum Jahre 2011 erreicht werden, dass ungefähr so viele Gelder in die neuen wie in die alten Mitgliedstaaten fließen. Ausgabenkategorie 2 „Nachhaltige Bewirtschaftung und Schutz der natürlichen Res- sourcen" bleibe hingegen in der Höhe stabil. Weiterhin erklärte Weise, dass die Ausgabenkategorien 3, die man vielleicht mit „Die EU für den Bürger" betiteln könnte, und 4, also „Die EU als globaler Partner“, in der Summe haushaltspolitisch nicht allzu brisant seien, auch wenn diese teilweise deutlich steigen würden.

Zur Nettozahlerproblematik im Spannungsfeld zwischen EU-Haushalt und der nationalen Haushaltslage wies Weise darauf hin, dass es näher liegend sei, auf die Nettosalden zu blicken, auch wenn sich die Bundesregierung auf die Bruttosalden fokussiert habe. Allgemein sehe man sich mit mindestens drei Problemfeldern konfrontiert, nämlich wie man Nettosalden erfassen, messen und schließlich auch bewerten solle. Dabei könne man im Hinblick auf die Erfassungsproblematik etwa die traditionellen Eigenmittel, die Agrarabschöpfungen und die Verwaltungsausgaben nicht sinnvoll berücksichtigen. Auch sollte man daran denken, dass mit den Nettosalden lediglich eine Zahlungsinzidenz ausgedrückt werden könne, aus ökonomischer Sicht jedoch die Frage nach den tatsächlichen Wirkungsinzidenzen der Ausgaben im Mittelpunkt stehen sollte. Da das Nettozahlerkonzept aber in den letzten Jahren immer populärer geworden sei und auch die Europäische Kommission mittlerweile die Argumentation aufgegriffen habe, stelle sich nun die Frage, wie Nettosalden in sinnvoller Weise gemessen werden könnten. Dabei seien Angaben in absoluten Größen wenig befriedigend. Sinnvoller sei es vielmehr, Nettosalden entweder in Pro-KopfAngaben oder als Anteil am nationalen Bruttoinlandsprodukt auszuweisen. Schwieriger zu beantworten sei allerdings die Frage nach der Bewertung der ermittelten Nettozahlerposition, da ein Vergleich der Nettosalden in gewisser Weise der Funktion des EU-Haushalts widerspräche, stelle dieser doch keinen bloßen Transfer von armen zu reichen Ländern dar. So sei zum Beispiel die Agrarpolitik von ihrer Grundanlage her ein Verteilungsinstrument zu Gunsten wirtschaftlich schwächerer Mitgliedstaaten. 
Als Konsequenz aus der Diskussion über die Nettosalden habe die Europäische Kommission bereits einen allgemeinen Korrekturmechanismus, aufbauend auf dem so genannten Briten-Rabatt, vorgeschlagen, da man der Ansicht sei, dass das bestehende System aus zwei Gründen nicht mehr zeitgemäß sei. Erstens habe seit der Gewährung des Briten-Rabatts eine relative Wohlstandsverschiebung zu Gunsten des Vereinigten Königreichs stattgefunden. Zweitens bestehe ein Konflikt zwischen der Tatsache, dass Großbritannien nicht vollständig an den Kosten für die Erweiterung beteiligt sei, gleichzeitig aber die neuen Mitgliedstaaten den Briten-Rabatt mit zu finanzieren hätten. Die politische Wirkung eines solchen Korrekturmechanismus sei jedoch nicht eindeutig vorhersehbar. Dieser könne zu einem Zuwachs an ausgabenintensiven Politiken führen, da mehr Mitgliedstaaten in die Finanzierung des EU-Haushalts miteinbezogen würden. Es könne daraus aber auch ein geringerer Reformdruck resultieren, da die Länder mit bisherigen Spitzenbelastungen zukünftig von Haushaltsbeschlüssen nicht mehr voll getroffen würden. Dies sei jedoch Spekulation und das Ergebnis derzeit noch nicht abzusehen.

Zum Spannungsfeld zwischen dem nationalen und dem EU-Haushalt erklärte Weise, dass der Finanzierungsanteil Deutschlands am EUBudget in Prozent des Bruttonationaleinkommens durch den Vorschlag der Kommission relativ stabil bleibe. Es sei jedoch die Frage nach der Dynamik, das heißt der Entwicklung der Haushalte zu stellen. So zitiere der Europaabgeordnete Böge in einem Bericht des Europäischen Parlaments einen durchschnittlichen Anstieg der Haushalte der Mitgliedstaaten im Zeitraum von 1996 bis 2002 um real 22 Prozent. Dagegen sei der Haushalt der Europäischen Union in der gleichen Periode lediglich um acht Prozent angewachsen. Langfristig sei deutlich zu erkennen, dass die Differenz zwischen den Wachstumsraten des Bruttoinlandsprodukts und der Ausgaben der Europäischen Union stetig zunehme.
Der deutsche Nettosaldo habe, je nach Berechnungsmethode, im Jahr 2003 bei 0,36 beziehungsweise 0,41 Prozent und der Bruttobeitrag bei 0,9 Prozent des Bruttonationaleinkommens gelegen. Die Staatsquote betrug im Jahr 2003 48,8 Prozent und das Haushaltsdefizit durchschnittlich 3,8 Prozent. Die Höhe der Staatsquote der EU-15 belief sich dagegen im Jahr 2003 im Durchschnitt der Mitgliedstaaten auf 48,5 Prozent und das Defizit im Euroraum auf 2,8 Prozent. Das Defizit Deutschlands ohne den Nettobeitrag hätte ohne die Abführungen an Brüssel immer noch 3,4 Prozent betragen.

Abschließend merkte Weise an, dass Politik in erster Linie mittels eines Bottom-up-Ansatzes zu bewerten sei, man sich also die Einzelpolitiken anschauen sollte, um die Frage nach deren effizienter Gestaltung beantworten zu können. Daneben müsse Politik natürlich auch aus einer Top-down-Perspektive bewertet werden, um eine gewisse finanzielle Schmerzgrenze nicht zu überschreiten. Allerdings werde momentan zu viel über die reine Ausgabenobergrenze und zu wenig über die tatsächlichen Politiken geredet. Auch seien mögliche Einsparpotenziale für ihn unklar. So seien die Agrarausgaben bereits auf mehrere Jahre hinweg festgelegt und die Strukturfondszahlungen an die neuen Mitgliedstaaten politisch nur schwer antastbar, wodurch im Wesentlichen nur noch die Strukturausgaben an die alten Mitgliedstaaten oder die so genannten Lissabon-Politiken für substanzielle Kürzungen übrig blieben.

Den Hintergrund der Ausführungen von Friedrich Heinemann bildeten am Zentrum für Europäische Wirtschaftsforschung durchgeführte Berechnungen, die es erlaubten, einige der derzeit im Hinblick auf die Ausgestaltung der Finanziellen Vorausschau diskutierten Optionen zu bewerten. In seinem Vortrag standen das Verhältnis der nationalen Haushaltspolitik zum EU-Budget sowie die Verteilungswirkungen und damit verbundene politische Optionen im Zentrum der Untersuchung. So wies Heinemann darauf hin, wie 
auffällig parallel die Fronten und Mehrheitsverhältnisse für verschiedene Reformvorschläge zur Trennlinie zwischen Nettozahlern und -empfängern verlaufen würden. Ungeachtet möglicher normativer Kritik an dem Konzept, sei es als Faktum anzusehen, dass die Mitgliedstaaten ihre Position entlang dieser Linie definierten und damit die Frage nach der Verteilungswirkung sehr bedeutend bleibe.

Das normative Ziel einer Maximierung des europäischen Mehrwerts sei sehr misstrauisch zu betrachten, wenngleich dies der Schlüssel zur Bewertung einer Aufgabenverlagerung von der nationalen auf die europäische Ebene sei. Die historische Erfahrung zeige jedoch, dass die Entscheidungen in der Regel von den Verteilungswirkungen bestimmt wurden und nicht vom Ziel einer Maximierung des europäischen Mehrwerts. So hätten verschiedene Studien nachgewiesen, dass große Staaten ihre relative Macht zu einer Erhöhung ihrer Nettosalden eingesetzt hätten und diese sozusagen ein Proxy für nationales Interesse seien. Da das EU-Budget sehr stark verteilungsorientiert bleibe, sei auch die Frage nach dem Nettosaldo weiterhin entscheidend zur Bewertung einzelner Politiken. In einem System von fiskalisch unitarischen Staaten, die keine Präferenz hätten, an welche Ebene das Geld zurückfließt, wäre der Nettosaldo sicherlich der einzig relevante Indikator. Da diese Bedingungen jedoch nicht erfüllt seien, blieben Bruttobeiträge auch weiterhin wichtige Indikatoren.

Betrachte man den von der Europäischen Kommission vorgelegten Vorschlag zur Finanziellen Vorausschau 2007 bis 2013 unter dem Gesichtspunkt der durchschnittlichen Pro-Kopf-Belastungen, so werde deutlich, dass die größten Verlierer Länder wie Irland und Spanien sein würden. Der Aussage von Weise, das EU-Budget stehe nicht im Konflikt mit der nationalen Haushaltskonsolidierung, widersprach Heinemann. So sei zum Beispiel der Bundeshaushalt strukturell problematisch unterfinanziert. Dies zeige allein, dass die Ausgaben nur zu 75 Prozent von Steuereinnahmen gedeckt seien. Vor diesem Hintergrund sei jeglicher Anstieg des Bundeshaushalts kritisch zu betrachten. Führe die Bundesrepublik derzeit noch rund zehn Prozent der Steuereinnahmen an die Europäische Union ab, so würde man gegen Ende der nächsten Finanziellen Vorausschau bereits bei 13 Prozent anlangen.

Die größten Nutznießer des Vorschlags der Europäischen Kommission wären Frankreich, Dänemark und die Niederlande. Eine vollständige Abschaffung des Briten-Rabatts wäre für Großbritannien mit einer jährlichen Mehrbelastung in Höhe von 6,3 Milliarden Euro verbunden. Die großen Gewinner einer Abschaffung wären dagegen Frankreich und Italien. Aus deutscher Sicht würde hingegen der allgemeine Korrekturmechanismus eine attraktivere Variante darstellen, da Deutschland den Briten-Rabatt derzeit nicht vollständig mitfinanziert.

Abschließend prognostizierte Heinemann eine gute Chance für die Realisierung der Einprozent-Grenze, bezeichnete die Aufhebung des Briten-Rabatts dagegen eher als unrealistisch. Vielmehr seien Kürzungen in der Kohäsionspolitik zu erwarten.

Reform des Eigenmittelsystems der Gemeinschaft

Peter Becker untersuchte in seinem Beitrag den Vorschlag der Europäischen Kommission zur Reform des Eigenmittelsystems in Hinblick auf mögliche Mehrheitsverhältnisse im Rat beziehungsweise dessen Realisierungschance. Der Kommissionsvorschlag gehe dabei auf das Jahr 2000 und die Abfassung der Lissabon-Strategie zurück, aus der die Kommission auch den Handlungsbedarf abgeleitet habe. Nach dem Bericht über das Funktionieren des Eigenmittelsystems habe sich das gegenwärtige System grundsätzlich bewährt, allerdings genüge es nicht den Kriterien der Transparenz und der Einfachheit. So fehle die Finanzautonomie von den Beiträgen der Mitgliedstaaten und Transparenz hinsichtlich der 
Summe, die der Unionsbürger für den Haushalt aufbringen müsse, und der Leistung die er dafür erhalte. Die Europäische Kommission komme zu dem Ergebnis, dass sich die Diskrepanz zwischen der wirtschaftlichen Leistungsfähigkeit und den Zahlungsverpflichtungen insbesondere der großen Nettozahler verstärke. Der direkte Vergleich der Wohlstandsentwicklung Deutschlands und Großbritanniens im Vergleich zu den übrigen Mitgliedstaaten zeige, dass der Wohlstand in Deutschland relativ gefallen und in Großbritannien gestiegen sei. Daher gebe es ein offensichtliches Missverhältnis zwischen der Haushaltslage und -belastung einiger großer Nettozahler. Auf dieser Problemanalyse aufbauend habe die Kommission zwei Vorschläge unterbreitet: die Einführung eines allgemeinen Korrekturmechanismus sowie eine steuerliche Eigenmittelquelle. Der allgemeine Korrekturmechanismus solle dabei durch die Einführung eines Schwellenwerts als eine Art Sicherheitsnetz für die großen Nettozahler dienen. Damit orientiere sich die Kommission grundsätzlich an dem System des Briten-Rabatts, wobei der allgemeine Korrekturmechanismus prinzipiell jedoch allen Mitgliedstaaten zu Gute käme. Die vorgeschlagenen Übergangsregelungen sollen eine allmähliche Eingliederung Großbritanniens und der anderen Mitgliedstaaten in das System des neuen Korrekturmechanismus bis zum Jahr 2011 erleichtern. Darüber hinaus schlage die Europäische Union vor, die Mehrwertsteuer-Eigenmittelquelle zu teilen und ein Prozent der Einnahmen direkt an den Unionshaushalt abzuführen. Die vorgeschlagene steuerliche Einnahmequelle solle dabei nicht an die Stelle von bisher existierenden Einnahmequellen treten, sondern diese ergänzen.

Bei den Verhandlungen im Ministerrat stünden der allgemeine Korrekturmechanismus sowie der Briten-Rabatt, die auf der Einnahmeseite zusammen behandelt würden, im Mittelpunkt. Dabei würden sich andere Koalitionsbildungen abzeichnen, als man sie auf der Ausgabenseite beobachten könne. Der Vorschlag zur Einführung des allgemeinen Kor- rekturmechanismus spalte sowohl die Nettozahler als auch die Nettoempfänger. Auf der Seite der Nettozahler sei, neben Großbritannien, vor allem die Dreiergruppe Deutschland, Schweden und die Niederlande zu finden, die ein eigenes ,non-paper ' vorgelegt habe, in welchem sie vor allem die Verbindung von Ausgaben- und Einnahmeseite herzustellen versuche. Frankreich und Österreich würden, so wie einige Nettoempfänger-Länder, dagegen jeden Rabatt oder Korrekturmechanismus ablehnen. Darüber hinaus hätten sich Spanien, Ungarn, die baltischen Staaten und Tschechien gegen einen Korrekturmechanismus ausgesprochen.

Die Folgen des allgemeinen Korrekturmechanismus für die Nettozahler seien eine Nivellierung der Unterschiede in den Nettozahlerpositionen, wodurch das System an Gerechtigkeit gewinnen würde. Durch eine mögliche Ablösung der Mehrwertsteuer-Eigenmittelquelle durch eine vollständige Bruttonationaleinkommen-Eigenmittelquelle würde Dänemark relativ, mit einen Minus von 2,66 Prozent des Bruttonationaleinkommens, und Italien absolut, mit einem Minus von rund 2,5 Millionen Euro, am meisten verlieren. Für die Bundesrepublik wäre eine derartige Umstellung auf eine vollständige Finanzierung des EU-Budgets über Bruttonationaleinkommen-Eigenmittel mit einem Verlust in Höhe von 180 Millionen Euro verbunden. Jedoch sei dies eher ein Randthema der derzeitigen Verhandlungen.

Abschließend äußerte Becker die Befürchtung, die Verhandlungen könnten an der Frage des Briten-Rabatts und dem allgemeinen Korrekturmechanismus scheitern. Die englische Regierung könnte aber ein Interesse daran haben, die Agenda 2007-Verhandlungen nicht mehr als Ballaststoff mit in ihre eigene Ratspräsidentschaft im zweiten Halbjahr 2005 zu nehmen. Dadurch öffne sich ein kleines Zeitfenster Ende Mai bis Anfang Juni, in dem eventuell ein Kompromiss gefunden werden könnte. Jedoch müsse dies eine Lösung sein, die vor allem auch im Hinblick auf 
ein mögliches Referendum über den europäischen Verfassungsvertrag den Wählern in Großbritannien als Erfolg verkauft werden könnte. Wahrscheinlich sei bei alldem keine umfassende Reform des Systems sondern eine europäische Kompromisslösung, die an mehreren Stellen Änderungen vornimmt. Die Einführung eines allgemeinen Korrekturmechanismus sei aus deutscher Sicht sicherlich wünschenswert, jedoch nicht aus europäischer, da damit ein Systembruch im Eigenmittelsystem verankert werden würde. Unbedingt zu verhindern seien zwei unterschiedliche Rabattsysteme. Ideal wäre es laut Becker, die Einführung eines allgemeinen Korrekturmechanismus mit der Reform des Eigenmittelsystems zu verbinden, um somit den Systembruch, der durch den allgemeinen Korrekturmechanismus entstehen würde, zu kompensieren.

Peter Schönberger wies in seinem Beitrag darauf hin, dass, würden die Ausgaben der Europäischen Union der Vergangenheit betrachtet werden, man den Eindruck erhalte, dass die Augen oftmals ,größer als der Mund und sicher größer als der Magen" gewesen seien. Für das Jahr 2003 seien Ausgaben in Höhe von 102,76 Milliarden Euro vorgesehen gewesen. Tatsächlich seien jedoch nur 90,558 Milliarden Euro ausgegeben worden, und damit zehn Prozent weniger als vorhergesehen. Deswegen sei es auch ein strategischer Fehler der Europäischen Kommission gewesen, sich nahezu ausschließlich auf die Neugestaltung der Ausgaben zu konzentrieren und die Reform der Einnahmeseite praktisch auszuklammern.

Im besten Falle würden der Verfassungsvertrag und ein neuer Beschluss über das Eigenmittelsystem zeitgleich in Kraft treten, um sicherzustellen, dass die im Verfassungsvertrag formulierten Ziele auch stabil finanziert werden können. Dabei werde in diesem von dem Willen der Bürgerinnen und Bürger und der Staaten Europas gesprochen, ihre Zukunft gemeinsam zu gestalten. Dadurch werde klar, dass sich die Finanzierung der Europäischen
Union zukünftig auf zwei Pfeilern gründen solle. Diese Idee wurde von der Europäischen Kommission in ihrem Bericht über das Funktionieren des Eigenmittelsystems aufgegriffen. Erstrebenswert seien danach also Beiträge von Seiten sowohl der Bürgerinnen und Bürger als auch der Mitgliedstaaten. Leider, so auch im Bericht über das Eigenmittelsystem, finde man keinen wirklichen Vorschlag für eine Reform des Eigenmittelsystems, sondern nur eine unverbindliche Handlungsanweisung an die Europäische Kommission zur weiteren Prüfung des bestehenden Systems und zur Vorlage eines möglichen Reformpakets. Vorgeschlagen wurde dementsprechend auch nur ein verbesserter Status quo, das heißt die Beibehaltung des bisherigen Systems mit einer einzigen Neuerung, dem allgemeinen Korrekturmechanismus.

Das bestehende System gestalte sich dabei aus den folgenden Komponenten: den traditionellen Eigenmitteln, den so genannten Mehrwertsteuer-Eigenmitteln und den Eigenmitteln, die auf Grundlage der Bruttonationaleinkommen berechnet werden. In Zahlen ausgedrückt bedeute dies, dass im Jahr 2003 10,8 Milliarden Euro an traditionellen Eigenmitteln eingenommen wurden, 21,2 Milliarden Euro aus Mehrwertsteuer-Mitteln und 51,2 Milliarden Euro an Bruttonationaleinkommen-Mitteln. Historisch betrachtet habe die Bedeutung der Bruttonationaleinkommen-Eigenmittel stark zugenommen und liege für das laufende Jahr über der Grenze von zwei Dritteln. Auch sei anzunehmen, dass der Anteil der Bruttonationaleinkommen-Eigenmittel im gegenwärtigen System weiter steigen werde. Die traditionellen Eigenmittel, vor allem die Zölle, lassen sich dabei im Grunde nicht einzelnen Mitgliedstaaten zuordnen, sondern gehören der Europäischen Union, die ja gerade auch eine Zollunion sei. Allerdings werden die Zölle von den Dienststellen der Mitgliedstaaten erhoben, die sich dafür geradezu fürstlich entlohnen ließen. Seit dem Eigenmittelbeschluss des Jahres 2000 könnten diese pauschal 25 Prozent der erhobenen Zölle als so genannte Erhebungskosten einbehalten. Es 
sei ein offenes Geheimnis, dass seinerzeit diese Erhöhung der Pauschale von zehn auf 25 Prozent ein Rabatt insbesondere zu Gunsten der Niederlande und Belgiens war, die in der Regel mehr an traditionellen Eigenmitteln einnehmen als zum Beispiel Italien. Das Hauptrisiko bei den traditionellen Eigenmitteln bestehe in der Hinterziehung von Zöllen, entweder durch falsche Angaben bei der Deklarierung der Güter oder durch Schmuggel. Dieses Risiko lasse sich zwar nicht exakt beziffern, sei jedoch in der Summe erheblich und man müsse sich fragen, ob das gegenwärtige System ausreichende Anreize für die Mitgliedstaaten bereithalte, diesen Anteil zu minimieren. Des Weiteren stütze sich die Berechnung der Mehrwertsteuer-Eigenmittel zum Großteil auf makroökonomische Berechnungen, weshalb die Kommission von einer statistischen Mehrwertsteuer-Einnahme spreche. Die Kontrolle dieser Berechnungen sei auf Grund der Komplexität entsprechend schwierig und der Missbrauch schlage direkt auf die eigenen Mittel der Europäischen Union durch. Zwar würden die fehlenden Einnahmen durch die BruttonationaleinkommenEigenmittel aufgestockt, das Problem liege jedoch auch weiterhin in der fairen Lastenverteilung zwischen den Mitgliedstaaten. Die Möglichkeit einer reinen Finanzierung des Unionshaushalts durch Bruttonationaleinkommen-Eigenmittel hätte sicherlich den Vorteil der Einfachheit, würde aber eine höhere Verlässlichkeit der Zahlen voraussetzen. Leider lasse deren Qualität, wie jüngst der Fall des griechischen Defizits gezeigt habe, oftmals sehr zu wünschen übrig.

Zum Abschluss wies Schönberger auf die großen Vorteile hin, die Exportnationen, allen voran Deutschland, durch den Binnenmarkt und die Erweiterung genießen würden. Ein Ausweg aus dem Problem der Nettosalden könnte die Einführung einer EU-Steuer sein, zum Beispiel eine Kerosinsteuer. Der wichtigste Vorteil einer solchen Steuer läge darin begründet, dass diese nicht eindeutig den einzelnen Mitgliedstaaten zuzuordnen sei und zusätzlich einen normativen Nutzen hätte.
Rolf Caesar konzentrierte sich in seinem Vortrag auf das Thema einer eigenen Steuerkompetenz für die Europäische Union, was seit langem für Zündstoff und unterschiedlichste Meinungen sorgt. Den Hintergrund bildeten dabei nicht nur die Forderungen der Europäischen Kommission, sondern auch der Europäische Verfassungsvertrag. So könnte Art. I-54 VVE bei genauer Betrachtung als Einfallstor für eine selbstständige Besteuerungskompetenz dienen, ermögliche dieser doch die Einführung neuer Einnahmekategorien. Die harmlos anmutende Formulierung dieses Artikels ziele eindeutig auf eine eigene Steuerkompetenz ab. In diesem Zusammenhang betonte Caesar, dass sich das Finanzierungssystem der Europäischen Union deutlich von dem der Mitgliedstaaten unterscheide und die Finanzverfassung auch immer ein Spiegel der Staatsverfassung sei. Allerdings sei die Europäische Union jedoch kein Staat und daher auch von den Beiträgen der Mitgliedstaaten abhängig, wodurch die Entscheidungsautonomie der Kommission erheblich eingeschränkt sei. Natürlich hätten die Kommission und das Europäische Parlament ein starkes Interesse, sich von diesen Zwängen zu befreien, jedoch könne eine Erhöhung der Finanzautonomie auch negativ als eine Beseitigung der Schranken für die Bestimmung eigener Einnahmen verstanden werden. Vor diesem Hintergrund sei die Frage nach der Aufgabe einer Finanzverfassung zu stellen. Aus traditionell-ökonomischer Sicht solle eine gute Finanzverfassung eine effiziente Verteilung von Ausgaben und entsprechenden Einnahmen auf Staatsebene sicherstellen. Das ökonomische Modell der Suche nach einer effizienten Mittelverteilung sei jedoch durch eine polit-ökonomische Sichtweise zu ergänzen, die Effizienz ganz anders definiere. So richte diese Sichtweise ihr Augenmerk nicht auf die allokative, sondern auf eine konstitutionelle Effizienz.

Anschließend diskutierte Caesar die Frage, ob es wohlfahrtsökonomische Gründe für eine Steuererhebungskompetenz der Europäischen Union gebe. Als zentrales Argument werde 
häufig die fiskalische Äquivalenz genannt, also die Übereinstimmung von Nutzenempfängern und Entscheidungs- und Finanzierungsträgern. Für die Europäische Union werde dabei argumentiert, dass die fiskalische Äquivalenz nicht gegeben sei, da die Entscheidungen über die Ausgaben und die Einnahmen getrennt gefällt werden würden. Eine Steuererhebungskompetenz, so die Argumente einiger Wissenschaftler, würde die Entscheidung über die Einnahmen besser mit der über die Ausgaben verknüpfen und damit die fiskalische Äquivalenz verbessern. Dies könne aber auch durch eine Beitragsfinanzierung erreicht werden. Darüber hinaus sei der Haushalt der Europäischen Union zu 80 Prozent ein Umverteilungshaushalt, für den das Argument der fiskalischen Äquivalenz gerade nicht gelte. Aus wohlsfahrtsökonomischer Sicht sei seiner Meinung nach mit Ausnahme der Zölle eine Steuererhebungskompetenz nicht erforderlich.

Die politischen Argumente für eine EUSteuer bezeichnete Caesar als nicht überzeugend, würde eine solche Steuer doch zum Beispiel den Steuerwettbewerb einschränken. Auch sei eine mögliche Lockerung der Budgetbeschränkung kritisch zu betrachten. Entgegen den Argumenten der Kommission würde außerdem die Einführung einer EUSteuer mit Sicherheit ein zusätzliches Aufkommen bedeuten. Sein Fazit aus wohlfahrtsökonomischer Sicht lautet daher, dass Zölle als EU-Steuern als sinnvoll zu betrachten seien, weitere Steuern beim derzeitigen Integrationsstand jedoch nicht wünschenswert wären, solange es keine andere Art von EUAktivitäten in Form europaweiter öffentlicher Güter gebe.

Reform der Strukturpolitik: ein Beitrag zur EU-Wettbewerbsfähigkeit?

Ingeborg Tömmel beleuchtete in ihrem Vortrag in erster Linie die Strategie der Kommission, die Strukturpolitik in die LissabonAgenda einzubeziehen. Bei der Zielvorstellung der Strukturpolitik falle auf, dass diese völlig anders als zuvor definiert werde. So solle die Strukturpolitik zukünftig den drei prioritären Zielsetzungen Konvergenz, Wettbewerbsfähigkeit und Kooperationen dienen. Als einziges inhaltlich relevantes Ziel bleibe jedoch nur das der Wettbewerbsfähigkeit übrig, welches unschwer als Kernziel der Lissabon-Agenda zu erkennen sei. Damit zeige sich bereits in der allgemeinen Zielformulierung, dass es der Europäischen Kommission bei der Reform der Strukturpolitik um die Verwirklichung der Lissabon-Agenda gehe. Durch den Versuch, viele einzelne Politiken zu bündeln und mit einem einzigen Ziel zu verbinden, betreibe die Kommission die Wiederbelebung eines integrativen Großprojekts, das mit dem Binnenmarktprogramm vergleichbar sei. Jedoch fehle diesem Projekt, anders als beim Binnenmarktprogramm, die Unterstützung der Mitgliedstaaten. So liege das Problem der Lissabon-Strategie darin, dass die Kommission eigentlich keine Kompetenzen zu deren Umsetzung besäße und die Mitgliedstaaten diese bisher nur sehr zögerlich umgesetzt hätten.

Die Ziele der Lissabon-Strategie nähmen nicht nur in der allgemeinen Zielformulierung einen prominenten Platz ein, sondern würden sich wie ein roter Faden durch die gesamten konkreten Zielformulierungen sowie die Beschreibungen der einzelnen Maßnahmen ziehen. Am deutlichsten sei das Ziel 2 auf die Lissabon-Agenda hin umformuliert worden und heiße nun im Kommissionsvorschlag „Wettbewerbsfähigkeit und Beschäftigung“. Laut Aussage der Europäischen Kommission bestehe auch eine generelle Übereinstimmung von über 50 Prozent zwischen den Zielen der Strukturpolitik und der Lissabon-Strategie. Allerdings konstatiere die Kommission auch, dass es dabei erhebliche Disparitäten gebe. So könnten die wohlhabenden Regionen die Ziele von Lissabon mittels der Strukturpolitik bis zu 85 Prozent, die ärmeren Region diese Ziele nur zu 18 bis 33 Prozent umsetzen. Dadurch werde deutlich, dass die eigentlichen Adressaten der Strukturpolitik sehr viel weniger in der Lage seien, diese Ziele zu verwirklichen. Jedoch sei der Versuch, auch die Ziel 
1-Regionen in die Strategie einzubeziehen, als Versuch zu werten, diese auch in die ärmeren Regionen zu transportieren.

Grundsätzlich lasse sich ableiten, dass die Umsetzung der Lissabon-Strategie für die Kommission mehr als ein Lippenbekenntnis sei und dass sie Verbündete zur Umsetzung ihrer Vorstellungen suche. Schließlich sei die Kommission bereits dabei, Vorschläge für konkrete Umsetzungsmaßnahmen einzuholen und die entsprechenden Leitlinien zu formulieren. Tömmel schlussfolgerte, dass die Vorschläge zur Reform der Strukturpolitik eine von der Kommission bewusst eingeschlagenen Strategie zur konkreten Umsetzung der Lissabon-Agenda seien, wobei sie sich eines altbekannten Musters bediene, nämlich neue Politiken in eine bereits eingefahrene Implementationsschiene einzugliedern. So hätten zum Beispiel die Strukturfonds in den Achtzigerjahren dazu gedient, die Forschungs- und Technologiepolitik salonfähig zu machen und auch die europäische Beschäftigungspolitik sei in den Neunzigerjahren durch die Strukturfonds finanziert worden. Im Falle der Lissabon-Strategie gehe dies jedoch über die bisherigen Entwicklungen hinaus, da diese kein einzelnes Politikfeld, sondern eine breite Bündelung von Politikfeldern sei, die alle schon seit Jahren auf europäischer Ebene diskutiert, jedoch nie wirklich umgesetzt worden wären.

Thomas Wobben fokussierte sich in seinen Ausführungen auf die Kernfrage der zukünftigen Mittelausstattung der Strukturpolitik zum Erreichen der Lissabon-Ziele. Die Strukturfonds seien bereits in der Vergangenheit sehr stark auf die Verbesserung der Angebotsbedingungen in den Regionen ausgelegt worden und die Lissabon-Ziele damit bereits Teil der Strukturpolitik gewesen. Die wirklichen Stärken dieses Politikbereichs lägen darin, dass es einen Programmansatz gebe, der die Regionen zwänge, sich über die Verbesserung der Wettbewerbsbedingungen in der Region Gedanken zu machen und ein indikatorgestütztes Programmmanagement zu installieren, von dem man in der nationalen Regionalförderung noch weit entfernt sei. Im Kontext der innerdeutschen Debatte habe dieser Programmansatz dazu geführt, dass die Mittel nur für die Bereiche eingesetzt werden konnten, die konkrete Ziele verfolgten, wohingegen zum Beispiel beim Solidarpakt eine erhebliche Zweckentfremdung der Mittel stattgefunden habe. Auch müsse man sehen, dass sich insbesondere die ostdeutschen Länder wesentlich bewusster geworden seien, wie sie mit ihren Mitteln umgehen müssen und sich die indikatorgestützte Programmauswertung mittlerweile auf ganz andere Programme auswirke.

Bei der Aufstellung des Finanztableaus habe es innerhalb der Europäischen Kommission offenbar unterschiedliche Zielvorstellungen gegeben. So existierten tiefgreifende Unstimmigkeiten die Frage der Mittelallokation betreffend, das heißt, ob die Exzellenz oder die strukturschwächsten Gebiete gefördert werden sollten. Dies habe zu einer langen Vertagung der Debatte über die Zukunft der Strukturfonds geführt, da über wichtige Punkte keine Einigung erreicht werden konnte. Das Europäische Parlament versuche, seine Rolle in der Strukturpolitik zu vergrößern und spricht sich für die Beibehaltung der rigiden Finanzkontrolle aus. Auch zum Wachstumsanpassungsfonds seien bisher keine negativen Äußerungen von Seiten des Europäischen Parlaments verlautbart worden.

Von Seiten der deutschen Länder gebe es bisher keine direkte Positionierung zur Finanzausstattung, jedoch eine eindeutige Opposition zu dem geplanten Paradigmenwechsel im Ziel 2-Bereich. Wichtig sei weiterhin das Verhältnis zur Ziel 1- und Ziel 2-Förderung in den alten Mitgliedstaaten. Die kohäsionspolitischen Aufgaben seien in der erweiterten Union im Sinne der originären vertraglichen Zielsetzung nicht mehr gegeben. Im Kern gehe es dabei um die Hilfe zu Gunsten der Schwächsten außerhalb der Ziel 2-Förderung.

\section{Lissabon-Agenda und Agenda 2007}

Georg Licht bezeichnete den Ausdruck Wettbewerbsfähigkeit, so wie er in der Lissabon- 
Strategie vorkomme, als einen Kampfbegriff ohne eigentlichen ökonomischen Inhalt. Mittlerweile habe man jedoch mehrere konkretere Ziele festgelegt, die bis zur Halbzeitüberprüfung jedoch nur mit mäßigem Engagement verfolgt worden seien. Auch sei lange Zeit nicht gesehen worden, dass es deutliche Prioritätenkonflikte gebe. Die Diskussion um die Finanzielle Vorausschau habe gezeigt, dass im Prinzip alles der Lissabon-Strategie zugeordnet werden kann, was Zweifel an der Ernsthaftigkeit des Prozesses zulasse.

Dass die Lissabon-Strategie prinzipiell eine Fortsetzung der Binnenmarktstrategie ist, werde vor allem bei den ersten Punkten, der Reform der Produkt- und Dienstleistungsmärkte sowie der Beschleunigung des Weges in die Bildungsgesellschaft sehr deutlich. Schaue man sich die nationale Politik der vergangenen Jahre an, könne man feststellen, dass nahezu alle Politiken unter die sechs Ziele der Lissabon-Strategie fallen. Damit werde auch das Problem einer zu breiten Definition der Zielsetzung offensichtlich. Jedoch gebe es eine empirische Evidenz für den $\mathrm{Zu}$ sammenhang zwischen Produktivitätswachstum und den Investitionen in Forschung und Entwicklung. Beim Vergleich mit den USA falle dabei auf, dass das staatliche Engagement in diesem Bereich ähnlich stark ausgeprägt ist, der Unterschied also vielmehr durch die geringeren privatwirtschaftlichen Investitionen zu Stande komme. In der EU-15 herrsche hier eine deutlich geringere Intensität als in Japan und den USA, auch wenn die Ausgaben in den USA im letzten Jahr, wegen fehlender Investitionen in der Automobilbranche und dem Telekommunikationssektor, um eine Milliarde US-Dollar rückläufig waren. Die geringen Investitionen in diesem Bereich in Europa seien eine Folge falscher Regulierung gewesen, die kaum Anreize für neue Technologien und damit Investitionen in Forschung und Entwicklung geboten habe. Daher gehe es für die Politik weniger darum, öffentliche Ausgaben zu erhöhen, als vielmehr die Rahmenbedingungen für eine Erhöhung privater Forschungsausgaben zu schaffen. Dabei seien die Forschungsrahmenprogramme, deren Mittelausstattung vom 1 . bis zum 7 . Rahmenprogramm deutlich erhöht wurde und heute etwa sieben Prozent der nationalen Ausgaben für Forschung und Entwicklung betrage, ein direktes Instrument der Europäischen Kommission.

Auch wenn es grundsätzlich wünschenswert wäre, dass der Kommissionsvorschlag für die Finanzielle Vorausschau speziell im Bereich Forschung und Entwicklung umgesetzt wird, sei letztendlich die Frage nach der Effizienz entscheidend, also ob Ausgaben für Forschung und Entwicklung auf EU-Ebene tatsächlich gewinnbringend eingesetzt wären oder ob diese Gelder besser auf nationaler Ebene verausgabt werden sollten. Mit den Mitteln sollen nach dem Kommissionsvorschlag vor allem fünf große Leitlinien verfolgt werden. Der größte Anteil solle dabei in thematische Programme (vor allem Informationsgesellschaft, Transport und Biotechnologie) fließen. Weiterhin soll mit dem European Research Council ein neues Instrument zur Förderung der Grundlagenforschung eingerichtet werden. Die Erfahrung mit den früheren Forschungsrahmenprogrammen habe gezeigt, dass vor allem vorwettbewerbliche, grenzüberschreitende Forschung gefördert werde. Der Wettbewerb um die Forschungsgelder sei dabei kein reiner Exzellenzwettbewerb, sondern verfolge auch deutlich das Kohäsionsziel. Dies zeige sich am Beispiel der thematischen Forschungsgeldervergabe: Würden die Gelder nach dem alleinigen Kriterium der Exzellenz vergeben werden, so wäre für Deutschland ein vergleichsweise hoher Anteil im Automobilbereich und im Maschinenbau zu erwarten, der jedoch nicht festzustellen sei. Dies habe zur Folge, dass die Gelder oftmals weniger effizient genutzt würden, als bei einer Vergabe auf nationaler Ebene. Sehr deutlich sei auch, dass sich die Rückflüsse aus den Rahmenprogrammen sehr stark mit dem prozentualen Anteil der nationalen Beiträge am Haushalt der Union decken würden. Eine Begründung für eine Forschungsförderung auf EU-Ebene sei damit unklar, auch wenn es 
wichtige Gründe dafür gebe. Sinnvoll sei beispielsweise eine Kooperation bei internationalen Großprojekten, wie zum Beispiel in der Luft- und Raumfahrtindustrie. Ein weiterer Widerspruch bestehe in der großen Überschneidung mit der nationalen Förderung. Dies spiegele sich nicht nur in der thematischen Förderung wieder, sondern auch darin, dass oftmals die gleichen Unternehmen an den europäischen wie den nationalen Forschungsprogrammen teilnehmen würden. $\mathrm{Zu}$ sätzlich senke der hohe Koordinierungsbedarf auf europäischer Ebene die Effizienz des Mitteleinsatzes. Viele internationale Kooperationen im Forschungsbereich könnten ohnehin einfacher über direkte Kontakte als den Umweg der Europäischen Union bewerkstelligt werden.

Pascal Hector vertrat in seinem Beitrag vier Thesen zur Lissabon-Agenda und der Finanziellen Vorausschau. Zunächst sei die Lissabon-Strategie primär eine Sache der Mitgliedstaaten und des Privatsektors und nicht eine Sache der Europäischen Union. Darüber hinaus sei die Lissabon-Strategie nicht in erster Linie auf finanzielle Maßnahmen bezogen. Drittens sei die Lissabon-Strategie damit auch kein Begründungstatbestand für höhere Mittelansätze und viertens müsse man bei einem Mittelvolumen von 1,0 Prozent des BIP in der nächsten Finanziellen Vorausschau, vor allem im Hinblick auf die gemeinsame Agrarpolitik, realistisch bleiben und die politischen Rahmenbedingungen beachten, was die Mittelausstattung für die Lissabon-Strategie betreffe.

Schaue man sich die Entwicklung der Lissabon-Strategie genauer an, so falle auf, dass die Strategie von Anfang an eine Koordinierung nationaler Bemühungen gewesen sei. Die Idee sei demnach, in Bereichen ohne originäre EU-Zuständigkeit die Mitgliedssaaten dazu zu bringen, in einer bestimmten Richtung zusammenzuarbeiten. Im Grunde blieben es aber die Mitgliedstaaten, die entscheiden, was gemacht werde, so dass diese lediglich eine Selbstverpflichtung unter der Sekretari- atshilfe der Europäischen Union eingehen würden. Dies sei etwas völlig anderes als im Bereich der supranationalen Zusammenarbeit, in dem die Europäische Union Zuständigkeiten besitze und somit auch legislative Vorgaben machen könne. Der Ausgangspunkt der Lissabon-Agenda sei eben auch die Offene Methode der Koordinierung gewesen. In diesem Zusammenhang dürfe man auch den Privatsektor nicht vergessen, hänge der Erfolg der Lissabon-Strategie doch im Wesentlichen von dessen Engagement ab. Dies erhöhe sich jedoch nur, wenn die ordnungspolitischen Rahmenbedingungen richtig gesetzt würden. Dementsprechend gehe es bei der LissabonStrategie nicht darum, große Geldbeträge zu mobilisieren, sondern einen Rahmen für die ordnungspolitische Koordinierung dieser Maßnahmen zu schaffen. Darüber hinaus gelte der Top-down-Ansatz auch bei der Lissabon-Agenda. So stehe nur ein begrenztes $\mathrm{Ma} ß$ an Ressourcen zur Verfügung, für das im Vorschlag der Bundesregierung als Grenze 1,0 Prozent des Bruttoinlandsprodukts festgelegt sei. Man müsse also versuchen, diese zur Verfügung stehenden Ressourcen möglichst effizient einzusetzen. Es dürfte die Höhe der Mittel nicht von Bedürfnissen abgeleitet werden, seien letztere doch per Definition stets unendlich.

Die Umorientierung im Rahmen des bestehenden Finanzrahmens sei sicherlich positiv zu bewerten. So hätten die Agrarausgaben in den 1980ern noch 80 Prozent des EU-Haushalts ausgemacht. Diese lägen derzeit jedoch nur noch bei etwa 45 Prozent und würden am Ende der nächsten Finanziellen Vorausschau im Jahr 2013 auf etwa 35 Prozent des EUBudgets reduziert sein. Diese Entwicklung lasse sich analog auch für die Strukturpolitik beobachten. Diese Einsparungen dürften jedoch nicht an anderer Stelle in unproduktiver Weise verwendet werden, sondern sollten möglichst zu einer Ausgabenreduzierung führen. Darüber hinaus resultiere der Vorschlag der Bundesregierung aus der Überzeugung, dass 1,0 Prozent der Mittel des Bruttoinlandsprodukts ausreichend seien, um alles zu finan- 
zieren, was sinnvollerweise auf der Ebene der Europäischen Union geleistet werden muss. Dabei sei die Agrarpolitik kein unmittelbarer Verhandlungsgegenstand, sei diese doch bereits im Jahr 2002 in dem so genannten Brüsseler Kompromiss bis zum Jahr 2013 festgelegt worden, was den Weg für eine $\mathrm{Zu}$ stimmung Frankreichs zur Osterweiterung ebnete. Zusammenfassend wies Hector noch einmal darauf hin, dass eine Umorientierung, so wie sie im Zuge der Lissabon-Agenda stattfinde, zu begrüßen sei. Auch bedeute der 1-Prozent-Ansatz eine reale Steigerung, beinhalte dieser doch sowohl eine nominelle Steigerung als auch die Berücksichtigung künftigen Wirtschaftswachstums.

Raoul Wirtz verwies zunächst auf die Schlussfolgerungen des Europäischen Rates von Lissabon hin, die eindeutig festlegen würden, dass die Umsetzung des Lissabon-Prozess eine Sache des nationalen Willens sei. Andererseits gebe es in den Schlussfolgerungen den Hinweis, dass die Finanzielle Vorausschau angemessene Mittel zum Erreichen der Lissabon-Ziele bereitstellen solle. Ein spezifischer Punkt der Verhandlungen sei die Tatsache, dass zwei Verhandlungen gleichzeitig stattfänden. So werde neben der eigentlichen Finanziellen Vorausschau parallel über die gesetzlichen Verordnungen verhandelt, was 1999 nicht der Fall gewesen sei. Weiterhin erschwere die Tatsache, dass viele Programme, vor allem in der Rubrik 1a, mittlerweile in Übereinstimmung mit dem Europäischen Parlament beschlossen werden müssen, die Verhandlungen erheblich. Als Konsequenz werde sich der Rat in einem politischen Abkommen, sei es im Juni 2005 oder später, nicht auf absolute Zahlen für die einzelnen Teilrubriken festlegen, da das Europäische Parlament diese Zahlen im Nachhinein recht einfach zurückweisen könne. Auch würde es der institutionellen Logik widersprechen, würde der Rat versuchen, diese Teilrubriken sehr detailliert festzuschreiben. Dieser Aspekt sei wichtig, einerseits im Hinblick auf die Verhandlungen über die Finanzielle Vorausschau und andererseits hinsichtlich der Orientierung ver- schiedener Politiken. So werde zum Beispiel im Rat sehr engagiert über die Ziele und Rahmenbedingungen der Forschungspolitik diskutiert. Skeptisch äußerte sich Wirtz im Bezug darauf, dass es im Juni 2005 ein gesetzliches Abkommen zur Forschungspolitik geben werde. So habe die Europäische Kommission ihren Vorschlag erst kürzlich vorgelegt und die Arbeit im Rat erst jetzt angefangen. Sollte es wider Erwarten doch ein Übereinkommen im Juni geben, wären die Grundlinien und -prinzipien weiter unklar. Sicher sei, dass es kein politisches Abkommen auf legislativer Ebene geben könne, solange nicht der gesamte Finanzrahmen beschlossen ist.

Wirtz betonte, dass es zahlreiche Länder gebe, die den Kommissionsvorschlag zur Finanziellen Vorausschau unterstützen. Das im Jahre 2003 beschlossene Datum Juni 2005 für die Entscheidung, hatte vor allem das Ziel sicherzustellen, dass die neuen Programme pünktlich Anfang 2007 starten können. Achtzehn Monate seien nicht zu viel Zeit, da die Europäische Kommission erst nach einem Kompromiss über den Finanzrahmen mit der konkreten Planung der Programme anfangen könne. Man wolle die Verspätungen, die es bei der Agenda 2000 gegeben habe, dieses Mal soweit wie möglich verhindern. Dies sei vor allem das Anliegen der Kohäsionsländer. $\mathrm{Ob}$ dies machbar sei, scheint jedoch im Moment nur schwer vorherzusagen. Die Präsidentschaft habe mit der so genannten Verhandlungsbox ein Instrument entworfen, welches bereits 1994 sowie 1999 angewandt wurde. Auf der Basis dieses Dokuments versuche man, einen möglichen Kompromiss zu erarbeiten. Klar sei auch, dass ein Großteil der finanziellen Mittel in den Rubriken 1 und 2 liegen, diese jedoch nicht gleich behandelt werden könnten, da die Rubrik 2 die spezifische Entscheidung über die Agrarpolitik von 2002 umfasse, die praktisch unantastbar sei. Es sei ebenfalls richtig, dass verschiedene Delegationen, vor allem aus den Kohäsionsländern, die Unvereinbarkeit dieses Kompromisses mit einem Haushaltsetat von nur einem 
Prozent, kritisieren. Dies würde im Vergleich zu dem Vorschlag der Europäischen Kommission de facto einen erneuten Anstieg der klassischen Agrarpolitik bedeuten. Auf der anderen Seite müssen auch Einsparungen in der Strukturpolitik vorgenommen werden, auch wenn die hauptsächlichen Kürzungen wahrscheinlich in der Teilrubrik 1a vorgenommen würden. Den britischen Rabatt betreffend erklärte Wirtz, dass die Grundbedingungen des Rabatts von 1984 nicht mehr erfüllt seien und es unter den Regierungen der Mitgliedstaaten eine breite Kritik an diesem Instrument gebe. Durch den Einstimmigkeitszwang werde ein Kompromiss in dieser Frage jedoch erheblich erschwert, auch wenn Tony Blair am Ende der Verhandlungen wahrscheinlich nicht isoliert dastehen möchte.

\section{Fazit}

Während der Tagung wurde ein weiter Bogen von einer spezifischen Politikfeldanalyse bis hin zu tiefen Einblicken in die aktuellen Verhandlungen über die Finanzielle Vorausschau gespannt. Deutlich wurden vor allem die Konflikte zwischen normativen Zielvorstellungen einerseits und realpolitischen Restriktionen andererseits. Einen Kompromiss zwischen den verschiedenen Positionen $\mathrm{zu}$ finden, dürfte dabei einer Herkules-Aufgabe gleichen. So wollen die derzeitigen Nettozahler der Europäischen Union künftig weniger in den gemeinsamen Haushalt einzahlen und verweisen auf die schwierigen ökonomischen Rahmenbedingungen. Auch wird bemängelt, dass die Europäische Union ein Glaubwürdigkeitsproblem habe, wenn sie für Europa immer mehr Geld fordere, gleichzeitig aber die
Mitgliedstaaten erfolgreich verklagen würde, die wegen $\mathrm{zu}$ hoher Haushaltsdefizite gegen den Stabilitätspakt verstoßen. Dem gegenüber stehen die neuen Mitgliedstaaten mit der Forderung nach mehr Solidarität und vor allem mehr Geld aus den Brüsseler Fördertöpfen. Weiterhin wollen auch die Nettoempfänger unter den alten Mitgliedstaaten die finanziellen Mittel aus den Strukturfonds der Europäischen Union nicht verlieren. Auch Großbritannien scheint alles andere als bereit, den eigenen Beitragsrabatt aufzugeben. Vor dem Hintergrund der gestärkten Front der Europakritiker in Großbritannien wird es für die Kommission schwierig, den Rabatt ernsthaft in Frage zu stellen. Somit hatte Großbritannien durch die Ankündigung, die Verfassung durch ein Referendum ratifizieren zu lassen, seine Verhandlungsposition für die nächste Finanzielle Vorausschau gestärkt. Unabhängig von dem letztendlichen Umfang des Unionshaushalts scheint es geboten, die einzelnen Programme genau nach ihrer jeweiligen Effizienz zu untersuchen, wenngleich diese keinesfalls ausschließlich dem Exzellenz-Kriterium folgen, sondern auch in einem großen Maße dem Kohäsionsziel verpflichtet bleiben.

Welche Bedeutung den Verhandlungen über die Finanzielle Vorausschau 2007 aus Sicht der Bundesregierung zukommt, ist wohl daran abzulesen, dass sie mit Finnland die Ratspräsidentschaft getauscht hat, um 2006 nicht erneut in der Rolle der Ratspräsidentschaft verhandeln zu müssen. Es bleibt also weiterhin spannend, wie und wann die 25 letztendlich zu einem Kompromiss über den zukünftigen Finanzrahmen der Europäischen Union kommen werden. 